See discussions, stats, and author profiles for this publication at: https://www.researchgate.net/publication/344439287

\title{
A Novel Approximation of Security-Constrained Optimal Power Flow with Incorporation of Generator Frequency and Voltage Control Response
}

Article in IEEE Transactions on Power Systems · October 2020

DOI: $10.1109 /$ TPWRS.2020.3028427

CITATIONS

0

4 authors:

Iason Avramidis

Luxembourg Institute of Science and Technology (LIST)

11 PUBLICATIONS 21 CITATIONS

SEE PROFILE

Stavros Karagiannopoulos

ETH Zurich

24 PUBLICATIONS 137 CITATIONS

SEE PROFILE

Some of the authors of this publication are also working on these related projects:

Project Autonomous supply with thermal and electrical energy for eco-cities View project

Project Data-driven control design in Active Distribution Networks View project
READS

142

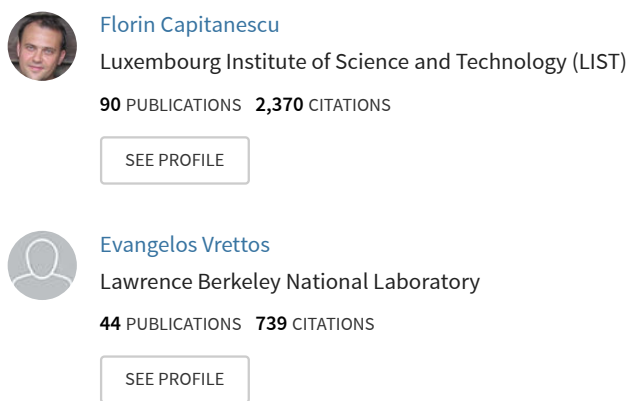




\title{
A Novel Approximation of Security-Constrained Optimal Power Flow with Incorporation of Generator Frequency and Voltage Control Response
}

\author{
Iason I. Avramidis, Student Member, IEEE, Florin Capitanescu, Stavros Karagiannopoulos, \\ Member, IEEE and Evangelos Vrettos, Member, IEEE
}

\begin{abstract}
This paper tackles key industry needs in the scope of the AC Security-Constrained Optimal Power Flow (SCOPF) framework, specifically two major challenging requirements regarding generators' post-contingency behavior: the (droop-based) frequency response and the voltage control mechanism (including PV/PQ switching). To this end, and to obtain a sufficiently accurate solution with a reasonable computational burden, we propose an approximation approach to SCOPF which extends significantly an existing algorithm with novel modelling elements: a continuous formulation to model generators' post-contingency responses based on successive approximations, a technique to evaluate the viability of contingencies with respect to the base case set-points, and the complexity reduction of all post-contingency states through network compression and power flow piecewise linearization. All new features are validated both separately and in unison, to monitor the improvement of the solution time and the potential degradation of the solution's quality. The proposed approach is successfully applied to three power system network models, the largest of which consists of 2,000 buses and more than 3,000 contingencies. Finally, the trade-off between the limitations in terms of solution quality and the benefits in terms of computational effort are assessed for all proposed approximations.
\end{abstract}

Index Terms-Contingency filtering, Generator contingency response, Mixed-integer nonlinear programming, Network compression, Security-constrained optimal power flow

\section{NOMENCLATURE}

A. Sets

$\begin{array}{ll}\mathcal{A} & \text { Set of areas } a \\ \mathcal{E} & \text { Set of lines } e \\ \mathcal{F} & \text { Set of transformers } f \\ \mathcal{G} & \text { Set of generators } g \\ \mathcal{I} & \text { Set of buses } i \\ \mathcal{K} & \text { Set of contingencies (0 corresponds to base case) } k \\ \mathcal{L} & \text { Set of generator cost segments } l \\ \mathcal{N} & \text { Set of slack penalty segments } n\end{array}$

\section{B. Parameters}

$$
\begin{array}{ll}
a_{g} & \text { Area of generator } g(-) \\
b_{i}^{F S} & \text { Bus } i \text { fixed shunt (FS) susceptance (p.u.) } \\
b_{e / f} & \text { Line } e, \text { transformer } f \text { series susceptance (p.u.) }
\end{array}
$$

For part of the work, the first and last author acknowledge the financial support of the Advanced Research Projects Agency - Energy (ARPA-E) of the US Department of Energy (award agreement number DE-AR0001075), within the scope of the Grid Optimization Competition (https://gocompetition.energy.gov/)

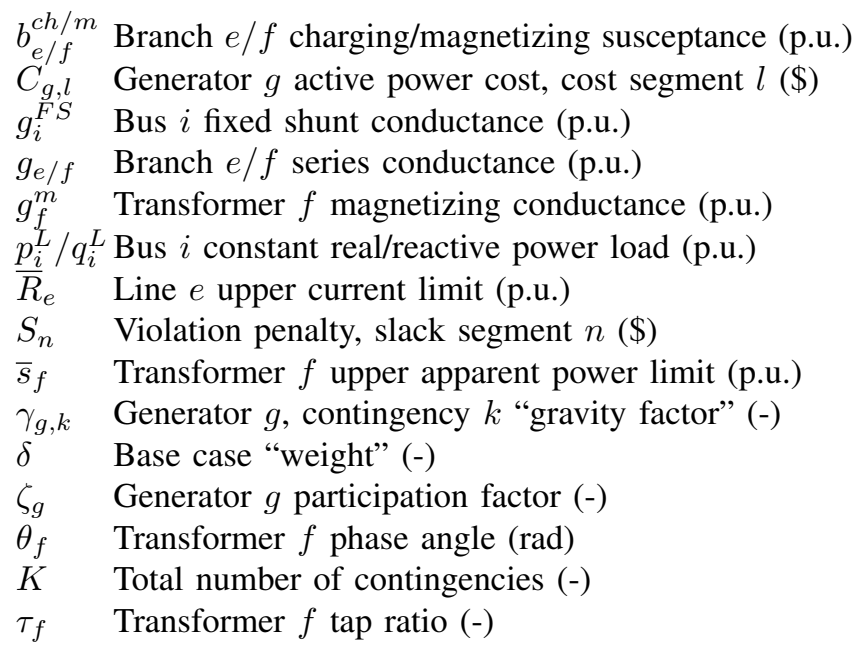

\section{Optimization variables}

$b_{i, k}^{C S} \quad$ Bus $i$, contingency $k$, contr. shunt susceptance (p.u.)

$p_{e / f, k}^{d, o}$ Branch $e, f$, contingency $k$, real power flow at destination/origin (p.u.)

$p_{g, k, l}$ Generator $g$, contingency $k$, real power output, belonging to cost segment $l$ (p.u.)

$q_{e / f, k}^{d, o}$ Branch $e, f$, contingency $k$, reactive power flow at destination/origin (p.u.)

$q_{g, k} \quad$ Generator $g$, contingency $k$, reactive power (p.u.)

$u_{i, k} \quad$ Bus $i$, contingency $k$ voltage magnitude (p.u.)

$\Delta_{k} \quad$ Contingency $k$ scale factor (p.u.)

$\theta_{i, k} \quad$ Bus $i$, contingency $k$ voltage angle (rad)

$\sigma_{e, k, n}^{I}$ Line $e$ apparent current slack, contingency $k$, segment $n$ (non-negative, p.u.)

$\sigma_{f, k, n}^{S}$ Transformer $f$ apparent power slack, contingency $k$, segment $n$ (non-negative, p.u.)

$\sigma_{i, k, n}^{P+}$ Bus $i$ active power overconsumption slack, contingency $k$, segment $n$ (non-negative, p.u.)

$\sigma_{i, k, n}^{P-}$ Bus $i$ active power underconsumption slack, contingency $k$, segment $n$ (non-negative, p.u.)

$\sigma_{i, k, n}^{Q+}$ Bus $i$ reactive power overconsumption slack, contingency $k$, segment $n$ (non-negative, p.u.)

$\sigma_{i, k, n}^{Q-}$ Bus $i$ reactive power underconsumption slack, contingency $k$, segment $n$ (non-negative, p.u.) 


\section{INTRODUCTION}

In the day-ahead operational planning of power systems, the AC security-constrained optimal power flow (SCOPF) is the most widely used tool for enforcing system security with respect to a set of (mainly $\mathrm{N}-1$ ) possible contingencies [1].

In general, a SCOPF calculates the optimal settings and/or re-dispatch of control means (e.g., generators' active power from market-cleared values) to enforce static constraints (branch loading and voltage profile) under normal, i.e., base case (BC) and post-contingency case (CC) operations. This is achieved through preventive [2] and/or corrective [3] measures. The number of variables (continuous and discrete), the control equipment variety and limitations and the nature of power systems (nonlinear, non-convex) render the SCOPF a computationally challenging problem. At its hardest, it is a mixed-integer nonlinear programming (MINLP) problem [4].

Most SCOPF research works consider only continuous variables (NLP problem), shifting their focus towards reducing the problem size [5]-[7]. To achieve this goal, some problem decomposition methods, aimed at the efficient identification of binding constraints in an iterative fashion, have been proposed based on: i) the progressive accumulation of constraints related to only potentially binding contingencies [6], [8]-[10], ii) Bender decomposition [3], [5], iii) the alternating direction method of multipliers [5], or iv) exploiting the problem structure of interior-point methods [7], [9]. Regardless of the approach, a common feature is solving an NLP problem at all outer loops of the decomposition, mostly by interior point algorithms [5]-[7], [9] or sequential linear programming [8]. Under the current state-of-the-art, only approximate solutions can be obtained for industrial SCOPF problems, which need to be solved within a reasonable time-frame. This requires approximated models of post-contingency states, obtainable via linearizations [1], [3], [5], [8] or network compression [6].

Another major challenge of SCOPF problems concerns the proper treatment of discrete variables [4]. When their number grows too large the problem faces tractability issues; even well-established MINLP solution methods (e.g., branch-andbound or outer approximation) are not viable. Techniques such as progressive rounding [6] or sophisticated heuristics have also been proposed (see [4], [11] for a comprehensive survey). While effective, they often produce sub-optimal or infeasible solutions. The challenge of how to properly handle discrete variables has been tackled to some extent for normal operating conditions, i.e., OPF problems [12], though few of the proposed techniques can be efficiently leveraged to SCOPF, where the challenge remains under-addressed.

While further computational improvements are certainly required, current state-of-the-art methods allow for (approximately) solving the large-scale deterministic AC SCOPF [5][7] in a reasonable time-frame. However, most works tend to address "academic" SCOPF formulations; practical gaps need to be bridged towards industrial adoption. Such is the case with the modelling of generators' response to contingencies; SCOPF methods proposed in academia rely on over- simplified assumptions such as: $(i)$ only one (slack) generator being responsible for primary frequency control and (ii) the proper modelling of voltage control being ignored (modelling the possibility of a generator exhausting its reactive power reserves would entail the use of challenging complementarity constraints [13], or even relaxing reactive power limits). It is clear that more realistic generator models are needed to better reflect the true requirements of SCOPF problems.

These issues have received little attention so far. For instance, works [14], [15] focus on heuristics for droop-based generators active power response to contingencies, based on a DC network model. A heuristic approach circumventing the use of complementarity constraints was proposed in [16] to manage the switching of generators between reactive limits exhaustion and undervoltage control states, though the approach is case-specific and exploits observations that are unique to heavy loading conditions. An attempt to address these issues was also recently launched in the form of the ARPA-E competition [17], which proposed a full, yet computationally burdensome SCOPF formulation as its research basis.

With SCOPF recently gaining some research momentum, on top of "conventional" approaches, new and highly specialized methods and problem formulations have been proposed within its scope, such as the sparse tableau representation of node breakers [18] or the cross-entropy method [19]. However, their validation and full potential have not been widely explored.

It becomes apparent that the addition of realistic modeling features significantly complicates an already challenging problem. This calls for practical approximation approaches, able to provide viable alternatives. Therefore, this work focuses on proposing a tractable solution approach to the most common version of the AC SCOPF problem (i.e., snapshot, deterministic) upgraded to address two major challenging requirements regarding the post-contingency behavior of generators: the (droop-based) frequency response and the voltage control mechanism (including PV/PQ switching).

The original contribution is a novel approach to solve the large-scale MINLP SCOPF, its new features being:

- A technique to handle PV/PQ generators switching in post-contingency via penalizing voltage slack variables.

- An iterative algorithm to model droop-based frequency regulation via successive NLPs which "relax and fix-tobound" the active power limits of generators. The original MINLP problem is thus reduced to a series of NLPs.

- An extension of the network compression technique (see [20]) to deal with binding interactions between the base case and the various contingency cases.

- A simple but effective heuristic to get rid of the overwhelming slack variables from the problem formulation.

- A significant adaptation of a previously proposed line flow convex approximations method [21], developed for $\mathrm{AC}$ OPF, which makes it for the first time applicable to the AC SCOPF problem.

The remainder of this paper is structured as follows. Section II presents the original SCOPF problem formulation. The proposed approximations are described in Section III. The 
solution algorithm is analyzed in Section IV. The results and validation of the proposed approximations are presented in Section V. Conclusions are outlined in Section VI.

\section{Preventive SCOPF Problem Formulation}

For the sake of paper self-containment, this section presents the problem formulation proposed by ARPA-E [17], for solving the AC SCOPF with advanced modelling features corresponding to industrial needs. Note that, in the Nomenclature, all variables are presented in their "full form", i.e., with all indices they may possibly require. However, if not essential for an equation, some indices are purposefully dropped for the sake of simplicity/saving space. The considered contingencies concern the failure of generators, lines and transformers.

\section{A. Objective function}

The objective function (1) is made up of the $\mathrm{BC}$ generation costs plus a weighted average of the $\mathrm{BC}$ and all CCs slack violation costs (penalty term), which are given in (2):

$$
\min \left\{\sum_{g \in \mathcal{G}, l \in \mathcal{L}} p_{g, 0, l} \cdot C_{g, l}+\delta \cdot c_{0}^{\sigma}+\frac{\sum_{k \in \mathcal{K} \backslash 0} c_{k}^{\sigma}}{|K| \cdot(1-\delta)^{-1}}\right\}
$$

where

$$
\begin{aligned}
c_{k}^{\sigma} & =\sum_{i \in \mathcal{I}, k \in \mathcal{K}, n \in \mathcal{N}} S_{n} \cdot\left(\sigma_{i, k, n}^{P^{+}}+\sigma_{i, k, n}^{P^{-}}+\sigma_{i, k, n}^{Q^{+}}+\sigma_{i, k, n}^{Q^{-}}\right) \\
& +\sum_{k \in \mathcal{K}, n \in \mathcal{N}} S_{n} \cdot\left(\sum_{e \in \mathcal{E}} \sigma_{e, k, n}^{I}+\sum_{f \in \mathcal{F}} \sigma_{f, k, n}^{S}\right) .
\end{aligned}
$$

Generation active power and slack violations are measured in terms of power and priced in a piecewise linear manner. Each generator adheres to its own price curve, while all slack violations (regardless of type) adhere to a common penalty curve. Readers are referred to [17] for the curve values.

\section{B. Nodal balances and branch flows}

The $\Pi$-equivalent transmission line model is used. The AC power flow equations (including fixed and controllable nodal shunt elements) are given in (3)-(4) $\forall i \in \mathcal{I}, \forall e \in \mathcal{E}, \forall k \in \mathcal{K}$ :

$$
\begin{gathered}
p_{e, k}^{d / o}=g_{e} u_{i_{e}^{d / o}, k}^{2}+\left[-g_{e} \cos \left(\theta_{i_{e}^{d / o}, k}-\theta_{i_{e}^{o / d}, k}\right)-\right. \\
\left.b_{e} \sin \left(\theta_{i_{e}^{d / o}, k}-\theta_{i_{e}^{o / d}, k}\right)\right] u_{i_{e}^{d}, k} u_{i_{e}^{o}, k} \\
q_{e, k}^{d / o}=-\left(b_{e}+\frac{b_{e}^{c h}}{2}\right) u_{i_{e}^{d / o}, k}^{2}+\left[b _ { e } \operatorname { c o s } \left(\theta_{i_{e}^{d / o}, k}-\right.\right. \\
\left.\left.\theta_{i_{e}^{o / d}, k}\right)-g_{e} \sin \left(\theta_{i_{e}^{d / o}, k}-\theta_{i_{e}^{o / d}, k}\right)\right] u_{i_{e}^{d}, k} u_{i_{e}^{o}, k}
\end{gathered}
$$

The transformer power flows are given by (5)-(6), adhering to modified versions of the AC power flow equations $\forall i \in$ $\mathcal{I}, \forall f \in \mathcal{F}, \forall k \in \mathcal{K}$, including magnetic elements, tap ratios and phase angles, when the power leaves the transformer $(o)$. When the power enters $(d)$, the tap-related terms are ignored:

$$
\begin{gathered}
p_{f, k}^{d / o}=\left(g_{f} \tau_{f}^{-2}+g_{f}^{m}\right) u_{i_{f}^{d / o}, k}^{2}-\left[\frac { g _ { f } } { \tau _ { f } } \operatorname { c o s } \left(\theta_{i_{f}^{d / o}, k}-\theta_{i_{f}^{o / d}, k}\right.\right. \\
\left.\left.-\theta_{f}\right)+\frac{b_{f}}{\tau_{f}} \sin \left(\theta_{i_{f}^{d / o}, k}-\theta_{i_{f}^{o / d}, k}-\theta_{f}\right)\right] u_{i_{f}^{d / o}, k} u_{i_{f}^{o / d}, k} \\
q_{f, k}^{d / o}=\left(b_{f} \tau_{f}^{-2}+b_{f}^{m}\right) u_{i_{f}^{d / o}, k}^{2}+\left[\frac { b _ { f } } { \tau _ { f } } \operatorname { c o s } \left(\theta_{i_{f}^{d / o}, k}-\theta_{i_{f}^{o / d}, k}\right.\right. \\
\left.\left.-\theta_{f}\right)-\frac{g_{f}}{\tau_{f}} \sin \left(\theta_{i_{f}^{d / o}, k}-\theta_{i_{f}^{o / d}, k}-\theta_{f}\right)\right] u_{i_{f}^{d / o}, k} u_{i_{f}^{o / d}, k}
\end{gathered}
$$

The nodal active and reactive power balance constraints are described by (7)-(8) $\forall i \in \mathcal{I}, \forall k \in \mathcal{K}$, also considering the various power balance violation slack variables:

$$
\begin{array}{r}
\sum_{g \in \mathcal{G}_{i}} p_{g, k}-p_{i, k}^{L}-g_{i}^{F S} u_{i, k}^{2}-\sum_{e \in \mathcal{E}_{i}^{o}} p_{e, k}^{o}-\sum_{e \in \mathcal{E}_{i}^{d}} p_{e, k}^{d}- \\
\sum_{f \in \mathcal{F}_{i}^{o}} p_{f, k}^{o}-\sum_{f \in \mathcal{F}_{i}^{d}} p_{f, k}^{d}=\sigma_{i, k}^{P^{+}}-\sigma_{i, k}^{P^{-}}
\end{array}
$$

$$
\begin{aligned}
& \sum_{g \in \mathcal{G}_{i}} q_{g, k}-q_{i, k}^{L}-\left(b_{i}^{F S}-b_{i}^{C S}\right) u_{i, k}^{2}-\sum_{e \in \mathcal{E}_{i}^{o}} q_{e, k}^{o}- \\
& \sum_{e \in \mathcal{E}_{i}^{d}} q_{e, k}^{d}-\sum_{f \in \mathcal{F}_{i}^{o}} q_{f, k}^{o}-\sum_{f \in \mathcal{F}_{i}^{d}} q_{f, k}^{d}=\sigma_{i, k}^{Q^{+}}-\sigma_{i, k}^{Q^{-}}
\end{aligned}
$$

\section{Technical limits}

The technical limitations in terms of voltage, generators' active and reactive output as well as the reactive injection of controllable shunts are given by (9)-(12), respectively:

$$
\begin{array}{r}
\underline{u}_{i, k} \leq u_{i, k} \leq \bar{u}_{i, k} \quad \forall i \in \mathcal{I}, \forall k \in \mathcal{K} \\
\underline{p}_{g} \leq p_{g, k, l} \leq \bar{p}_{g} \quad \forall g \in \mathcal{G}, \forall k \in \mathcal{K}, \forall l \in \mathcal{L} \\
\underline{q}_{g} \leq q_{g, k} \leq \bar{q}_{g} \quad \forall g \in \mathcal{G}, \forall k \in \mathcal{K} \\
\underline{b}_{i}^{C S} \leq b_{i, k}^{C S} \leq \bar{b}_{i}^{C S} \quad \forall i \in \mathcal{I}, \forall k \in \mathcal{K}
\end{array}
$$

Transmission lines and transformers are limited by current (13) and apparent power (14), respectively, $\forall i \in \mathcal{I}, \forall k \in$ $\mathcal{K}, \forall e \in \mathcal{E}, \forall f \in \mathcal{F}$ :

$$
\begin{gathered}
\sqrt{\left(p_{e, k}^{d / o}\right)^{2}+\left(q_{e, k}^{d / o}\right)^{2}} \leq \bar{R}_{e} \cdot u_{i_{e}^{d / o}}+\sigma_{e, k}^{I} \\
\sqrt{\left(p_{f, k}^{d / o}\right)^{2}+\left(q_{f, k}^{d / o}\right)^{2}} \leq \bar{s}_{f}+\sigma_{f, k}^{S}
\end{gathered}
$$

\section{Generators' contingency response}

1) Active power response: Assume that the out-of-service element corresponding to $\mathrm{CC} k$ belongs to area $A$. If a generator $g$ belongs to $A$ (affected area), it adjusts its active power output according to its offline participation factor $\zeta_{g}$ (until it reaches its technical limitations). Otherwise, it maintains its $\mathrm{BC}$ active output, as described by (15) [17]:

$$
p_{g, k, l}= \begin{cases}p_{g, 0, l} & a_{g} \neq A \\ p_{g, 0, l}+\zeta_{g} \cdot \Delta_{k} & a_{g}=A, p_{g, k, l} \in\left[\underline{p}_{g}, \bar{p}_{g}\right] \\ \underline{p}_{g} & a_{g}=A, p_{g, k, l} \leq \underline{p}_{g} \\ \bar{p}_{g} & a_{g}=A, p_{g, k, l} \geq \bar{p}_{g}\end{cases}
$$


When $a_{g}=A$, constraint (15) requires 2 binary variables per generator, indicating the generator has reached its upper or lower limit, "activating" the third or fourth line respectively. If neither of the binary variables is "active", the second line applies. Do note that it would be inaccurate to enforce a constraint of the type $\underline{p}_{g} \leq p_{g, 0, l}+\zeta_{g} \cdot \Delta_{k} \leq \bar{p}_{g}$, since that would limit the "flexibility" of $\Delta_{k}$, which should be allowed a wider range of values.

2) Reactive power response: When generator $g$ belongs to the affected area $\left(a_{g}=A\right)$, it tries to maintain its bus' $\mathrm{BC}$ voltage, until its reactive power reserves are exhausted [17]. This is referred to as generator PV/PQ switching and is described by (16). Two binary variables are required to indicate that the generator has reached its upper or lower limit, "activating" lines 2 or 3 respectively. If neither of the binary variables is "active", the nodal voltage magnitude has been successfully maintained (line 1):

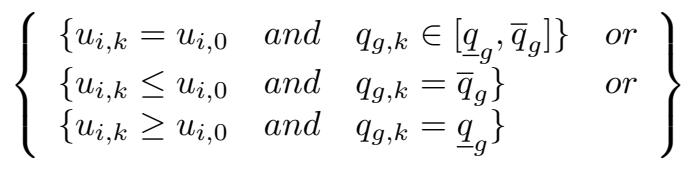

\section{Proposed Approximation ApProACH}

Even for small contingency sets, the large-scale MINLP SCOPF problem remains computationally challenging. The amount of slack variables (approximately twice the number of all "real" variables) coupled with the high number of binary variables (to model generators' response possible switching status) cannot be efficiently handled by conventional MINLP solvers. To remedy tractability concerns, the proposed approach contains several approximations described forthwith.

\section{A. Slack Removal}

Slack variables are necessary for large-scale SCOPF problems, preventing numerical issues if the original problem is infeasible. However, they prohibitively enlarge the problem size. As such, any slack variable that is reasonably expected to be zero at the optimum could instead be neglected.

We primarily focus on returning solutions with a physical meaning. As such, we neglect all slack variables that concern nodal power balances. These variables would only be active in cases of large generator outputs coupled with branches substantially overshooting their thermal limits. However, we assume that the power systems are well designed and such cases will not occur. We maintain the slack variables of the branches that are thermally loaded above a high degree in the BC. In this work, we define this threshold of thermal loading as $80 \%$ of their thermal limit. We assume that the slack variables of the remaining lines will remain inactive for all CCs. If the problem is infeasible, the slack removal limit can be relaxed.

\section{B. Handling generator responses}

Removing binary variables converts the problem to an NLP, reducing the computational complexity manifold. However, the problem approximation has to be sufficiently accurate.
1) Reactive response - Voltage slacks: Concerning the generator reactive power response, we begin by introducing two voltage-related slack variables per generator bus and contingency, $\sigma_{i, k}^{V^{d o w n}}, \sigma_{i, k}^{V^{u p}}$. Their "activation" is penalized in a linear fashion, the penalty being at least double the cost of the most expensive generator to produce its upper limit. We replace the original voltage constraints (16) with an approximation described by (17)-(18):

$$
\begin{aligned}
& u_{i, k}=u_{i, 0}+\sigma_{i, k}^{V^{u p}}-\sigma_{i, k}^{V^{\text {down }}} \\
& 0 \leq\left(\sigma_{i, k}^{V^{u p}}, \sigma_{i, k}^{V^{\text {down }}}\right)
\end{aligned}
$$

Since reactive power is not priced, the solver would first exhaust a generator's reactive output before resorting to the "activation" of a voltage slack variable. If an "activation" does occur at some bus, all connected generators have reached either their upper or lower reactive power limit, in which case the voltage deviates from its $\mathrm{BC}$ value. For generators outside the contingency area, they are not required to maintain their respective voltages; these slack variables are thus not needed.

2) Active response - Iterative NLP approximation: Concerning the generator active power response, all corresponding binary variables are initially disregarded, effectively transforming the problem to an NLP one. All CC generator active

\section{Algorithm 1: Combined active and reactive generator response approximation}

\section{Set $t=1$;}

2. Set $\underline{p}_{g, k}=-\infty \& \bar{p}_{g, k}=\infty \quad \forall g \in \mathcal{G}, \forall k \in \mathcal{K} \backslash 0$;

3. Define the set (per CC $k$ ) of "problematic" generators $\mathcal{Z}^{k}\left(\mathcal{Z}^{k}=\emptyset\right)$, where $\mathcal{Z} \subseteq \mathcal{G}$;

4. while $\exists g:\left\{p_{g, k}<\underline{p}_{g} \quad\right.$ or $\left.\quad p_{g, k}>\bar{p}_{g}\right\}$ or $t=1$

do

4.1. Solve approximated SCOPF;

4.2. Store the calculated results $\Delta_{k}^{*}$;

4.3. for $k \in \mathcal{K} \backslash 0$ do

if $\Delta_{k}>0 \quad \& \quad \exists g:\left\{p_{g, k}>\bar{p}_{g}\right\}$ then

a. Detect "problematic" gen. $\left\{g^{a}, \ldots, g^{n}\right\}$;

b. Update $\mathcal{Z}^{k}$ as $\mathcal{Z}^{k} \rightarrow \mathcal{Z}^{k} \cup\left\{g^{a}, \ldots, g^{n}\right\}$;

c. Set $p_{z, k}=\bar{p}_{g}$;

d. Enforce constraint $\Delta_{k}^{\min }=\Delta_{k}^{*}$ for $t+1$;

else if $\Delta_{k}<0 \quad \& \quad \exists g:\left\{p_{g, k}<\underline{p}_{g}\right\}$ then

a. Detect "problematic" gen. $\left\{g^{a}, \ldots, g^{n}\right\}$;

b. Update $\mathcal{Z}^{k}$ as $\mathcal{Z}^{k} \rightarrow \mathcal{Z}^{k} \cup\left\{g^{a}, \ldots, g^{n}\right\}$;

c. Set $p_{z, k}=\underline{p}_{g}$;

d. Enforce constraint $\Delta_{k}^{\max }=\Delta_{k}^{*}$ for $t+1$;

else

I CC $k$ is acceptable

end

end

4.4. Set $t=t+1$;

end

5. Calculated solution is acceptable 
power limits in (10) are removed and the SCOPF problem is solved, assuming full generator flexibility. If at the optimum, for all CCs, there are no "problematic" generators (over- or undershoot their original output limits), then the solution is considered an acceptable approximation of the MINLP problem. However, if there are CCs with "problematic" generators, emergency constraints are placed on these generators and the $\mathrm{CC}$ itself (in the form of constraining $\Delta_{k}$ ), and the problem is re-solved. The process is repeated until there are no more "problematic" generators. The combined approach (active and reactive response approximation) is presented in Algorithm 1.

3) Illustrative example: For the sake of clarity, we present a simple illustrative example of Algorithm 1, applied to the SCOPF of the 2-bus system presented in Fig. 1 (details in Table I). The considered contingency is the failure of $G_{1}$.

After solving the approximated SCOPF (step 4.1), at the base case, $G_{1}$ produces $100 \mathrm{MW}, G_{2}$ produces $50 \mathrm{MW}, G_{3}$ its minimum of $0 \mathrm{MW}$, and $\Delta^{*}$ is equal to $300 \mathrm{MW}$ (step 4.2). For the examined contingency (step 4.3), $G_{2}$ and $G_{3}$ are calculated as having to produce $120 \mathrm{MW}$ and $30 \mathrm{MW}$, respectively. The load is served, but $G_{2}$ is identified as problematic (step 4.3.a), added to the relevant set (step 4.3.b) and its production fixed at its upper limit of $100 \mathrm{MW}$ (step 4.3.c). The "missing" production will have to be made up by $G_{3}$, meaning $\Delta$ must be increased. Hence, the lower limit of $\Delta$ is set as $\Delta^{*}(300$ MW, step 4.3.d), and the iterator is increased (step 4.4).

When the above process is repeated, the base case solution remains as is, and $\Delta^{*}$ is re-calculated at $500 \mathrm{MW}$. For the examined contingency, $G_{2}$ and $G_{3}$ are re-calculated as having to produce $100 \mathrm{MW}$ (pre-fixed) and $50 \mathrm{MW}$, respectively. The load is served and no generator is problematic (operating out-

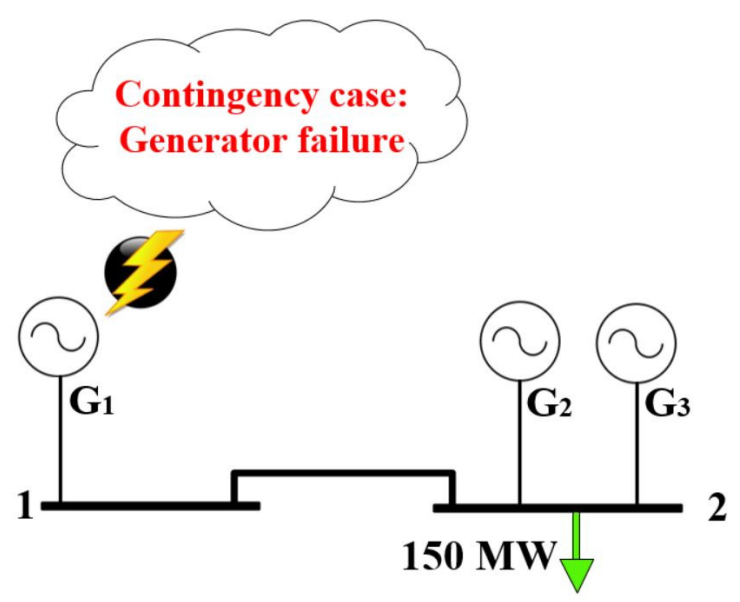

Fig. 1: Layout of 2-bus system example.

TABLE I: Characteristics of 2-bus system.

\begin{tabular}{|c|c|c|c|}
\hline & Limits (MW) & Costs (€/MW) & $\zeta$ \\
\hline$G_{1}$ & $0-100$ & 10 & 0.5 \\
\hline$G_{2}$ & $0-100$ & 20 & 0.233 \\
\hline$G_{3}$ & $0-100$ & 50 & 0.1 \\
\hline Line 1-2 & 500 & - & - \\
\hline
\end{tabular}

side its limits). As such, the solution is considered acceptable and the algorithm terminates.

\section{Network Compression}

It has been observed that contingencies have a limited effect on the power system, with only a small local area being affected in a substantial way [22], referred to as the "active" region. For the largely unaffected areas of the power system, collectively referred to as the "inactive" region, their postcontingency behavior is approximately the same as in the BC. This motivates shifting our focus to the affected area and using a simplified model for the remainder of the system.

For the active region, the original representation of all network elements is maintained. Contrariwise, the inactive region is replaced by an equivalent system of much smaller size. This is referred to as network compression. The borders of each area (per contingency) are defined by calculating the sensitivity of the $\mathrm{BC}$ voltages and power flows to the removal of a network element. The detailed equations can be found in [22], [23]. While there are several NC techniques, their performance within the scope of SCOPF is not well documented. In this work, the inactive region is represented by its radial equivalent independent (REI) [24], an approach that has seen some success in SCOPF problems [6].

A major challenge in applying $\mathrm{NC}$ in this work is the voltage control constraint (16), which binds the $\mathrm{BC}$ to all considered CCs. Since the NC process results in grouping together generator buses that belong to the inactive region, a direct application (per generator) of the aforementioned constraint is not straightforward. The following adaptation is proposed to address the issue.

Consider a group of $n$ generators $\mathcal{P}=\{1,2, \ldots, n\}$, connected to buses $\{1,2, \ldots, m\}$ ( $n \leq m)$ which will be compressed to the same bus $B$ post-contingency. The "gravity factors" $\gamma_{g}$ are defined for each generator $g \in \mathcal{P}$ as:

$$
\gamma_{g}=\frac{p_{g, 0}}{\sum_{g \in \mathcal{P}} p_{g, 0}}
$$

The original voltage control constraints are transformed to (20). This constraint is novel and also needs to be validated:

$$
V_{B}=\sum_{g \in \mathcal{P}} \frac{\gamma_{g} \cdot V_{g}}{n}
$$

\section{Branch Power Flow Linearization}

The lines and transformers for which the slack variables have been removed (see Section II) are assumed to operate without violating their power flow constraints for the entirety of the SCOPF problem. As such, it is proposed to simplify the problem by linearizing the power flow constraints of said branches. Each such constraint is replaced by a series of linear constraints (convex envelopes are created for each branch flow limit), representing different behavioral patterns concerning the branch voltage magnitudes and angles at the limit boundary. The utilized methodology is inspired from 
[21], and adapted to this work to address partial power system linearization and constraint differentiation between CCs. Performing this simplification for a high number of lines decreases the problem complexity of the problem and could lead to faster solution times. The reader is referred to [21] for an in-depth explanation of the linearization procedure.

\section{Iterative Solution Algorithm}

Even an NLP problem can be prohibitively large, leading to slow convergence and/or memory limitation issues. For example, the NLP approximation of the SCOPF concerning the Texas synthetic 2,000-bus system would require approximately 62 million variables and 142 million constraints. Therefore, there is strong motivation to further reduce the problem size and to cleverly manage the resulting problem.

This work is based on the multi-step solution algorithm developed in [6]. However, the approach is extended in several ways. The authors of [6] do not account for generator frequency response and voltage control; this work considers both by proposing a series of NLP approximations. Adaptations are also made to the security assessment (SA), and the treatment of potentially binding contingencies before adding them to the master problem. A contingency viability evaluation (CVE) module is added for contingencies that do not pass the SA (similar to a controllability evaluation, see [6], though extended to consider specific generator responses). The algorithm is presented in Fig. 2; each stage is hereby described.

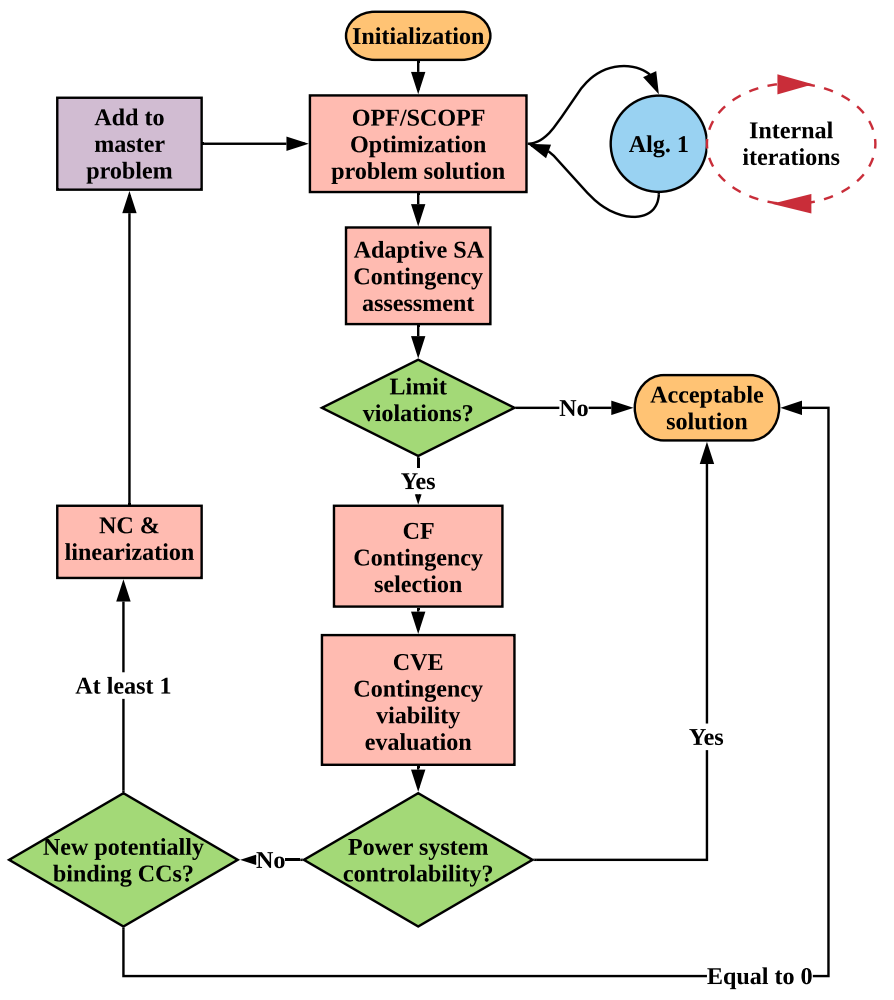

Fig. 2: SCOPF solution algorithm.
1) Initialization (IN): For interior point methods, the starting point significantly impacts the quality of the solution, in terms of speed, objective value and convergence [25]. The authors have explored various combinations of standard initialization options.

2) OPF/SCOPF: For the first iteration, no contingencies are considered $(k=0)$; this module thus refers to solving (using MATPOWER [26]) the OPF problem (1)-(14), albeit with some slacks removed and power flows linearized (Sections III.A, III.D). For subsequent iterations, by considering a reduced contingency set, a SCOPF problem (1)-(16) is solved (using IPOPT [27]). However, the following are modified:

a) Constraint set (15) is replaced by the proposed generator active response approximation (Alg. 1).

b) Constraint set (16) is replaced by (17)-(18).

c) Partial CC slack variables removal (Section III.A).

d) $\mathrm{CCs}$ added to the master problem are subject to NC (Section III.C) and linearization (Section III.D).

3) Adaptive SA: Based on the BC solution (OPF/SCOPF), we examine which contingencies lead to constraint violations through simple PFs (using MATPOWER). While straightforward for line outages, special provisions are made for generator outages, since the default system response would be incompatible with a successful SA. For a generator contingency $k$, if a loss of generation of $X$ MW can be addressed by setting $\Delta_{k}=X$ without violations, the $\mathrm{SA}$ is considered successful. Otherwise, a non-intuitive value of $\Delta_{k}$ is needed, and the SA is considered unsuccessful. The slack bus is dynamically defined when the contingency concerns the slack bus generator. Contingencies that fail the SA are called critical. If the set of critical contingencies is empty, the solution is acceptable and the algorithm terminates.

4) Contingency filtering (CF): There are several variations of $\mathrm{CF}$, the most prominent of which are presented in [9], [28]. This work utilizes the Individually Non-Dominated Contingency (INDC) scheme, based on the concept of "constraint violation domination". It consists of selecting the contingencies which lead to the largest violation of each constraint. These are the primary candidates to be added to the main SCOPF problem in the next iteration.

5) CVE: There are two possibilities concerning the filtered contingencies: either there is a value $\Delta_{k}$ that achieves acceptable operating conditions (we call these contingencies "viable") or not ("non-viable" contingencies). In this module, a modified OPF \{(3)-(14), (17)-(18), generator active response approximation $\}$, is solved for each, enforcing the generator response rules. A contingency is "viable" if a feasible solution can be returned. Non-viable contingencies guarantee unacceptable operating conditions and are included in the master SCOPF problem in order to calculate acceptable BC set-points (using an increased CC set). Non-viable contingencies are potentially binding for the problem. If the set of non- 
viable contingencies or the set of new potentially binding contingencies are empty, then the SCOPF problem has been sufficiently solved and the algorithm terminates.

6) NC \& Linearization (LIN): An optional NC module can be used to drastically reduce the size of the postcontingency system model that is added to the SCOPF problem. In our work, this is further complimented by a linearization technique, applied to some branch flow limits. These simplifications are applied to both the SCOPF and CVE modules.

Note: Due to the current stage of the work being methodological, the algorithm is applied in a serial manner; a parallel solution architecture could further improve its performance.

\section{NUMERICAL RESULTS}

\section{A. Test systems and problem size}

Since the majority of the proposed applications/extensions are novel, it is vital to validate them. As such, proof-of-concept applications are first executed in smaller systems, before transitioning to larger systems. Three transmission systems are used for validating and subsequently applying the proposed techniques: the IEEE 14-bus, the South Carolina synthetic 500bus and the Texas synthetic 2,000-bus systems [17]. A flat-start initialization is employed, unless otherwise stated.

\section{B. Performance of proposed techniques}

1) Iterative NLP approximation (SCOPF module): Validating the proposed approach is difficult, as it requires a comparison against solving exact SCOPFs. As such, our validation is contained to the 14-bus SCOPF (4 contingencies) and a smaller version of the 500-bus SCOPF (with 15 contingencies). The MINLP (using BONMIN [29]) and the proposed versions are compared, with results presented in Tables II, III.

For the 14-bus system (4 CCs, 5 generators), a few small active power mismatches are observed. There is an average mismatch of $0.045 \%$ per contingency, with the highest mismatch being $0.069 \%$. While not shown, the objective function is higher than the MINLP one, the difference being less

TABLE II: Validation of NLP approximation: 14-bus system.

\begin{tabular}{|c|c|c|c|}
\hline CC & Gen. & $P^{\text {MINLP }}(\mathrm{MW})$ & $P^{\text {Proposed }}(\mathrm{MW})$ \\
\hline 1 & 3 & 5.809 & 5.805 \\
\hline 1 & 4 & 11.471 & 11.465 \\
\hline 1 & 5 & 61.963 & 61.973 \\
\hline 2 & 3 & 5.809 & 5.805 \\
\hline 2 & 4 & 11.471 & 11.465 \\
\hline 2 & 5 & 61.935 & 61.945 \\
\hline
\end{tabular}

TABLE III: Validation of NLP approximation: 500-bus system.

\begin{tabular}{|c|c|c|c|}
\hline $\begin{array}{c}\text { Max Dev. } \\
(\%)\end{array}$ & $\begin{array}{c}\text { Avg. Dev. } \\
(\%)\end{array}$ & $\begin{array}{c}\text { Instances of } \\
\text { IVB (out of/930) }\end{array}$ & $\begin{array}{c}\text { Objective } \\
\text { diff. (\%) }\end{array}$ \\
\hline 0.312 & 0.073 & 923 & 0.47 \\
\hline
\end{tabular}

Dev: Deviation from MINLP generator active power output than $0.01 \%$ (with no guarantee of what formulation provides more accurate results). The proposed approach returned results within a few seconds, while the original MINLP formulation required approximately 500 seconds.

For the 500-bus system, the generator active power deviations from the MINLP case are small. The highest deviation across all 15 contingencies is $0.312 \%$. The average deviation per contingency is $0.073 \%$. Concerning the reactive power response of generators, the issues are minimal. In fact, out of the 930 voltages ( 15 contingencies $\times 62$ generators), there are only 7 instances where Identical Voltage Behaviour (IVB), i.e., same voltage value, is not observed between the MINLP and the proposed approach. However, the voltages that should be controlled are controlled. The objective function is $0.5 \%$ higher than the MINLP one (due to voltage slacks). The proposed approach returned results in less than 20 seconds, while the MINLP formulation required well over 3 hours.

2) Initialization: The initialization certainly impacts the solution speed. For example, after running several SCOPF test cases for all networks, it was observed that initializing angles and active powers using the DCOPF and voltage magnitudes using random values between 0.9 p.u. and 1 p.u. could increase the solution speed by up $90 \%$, as compared to a converged PF initialization. Several initialization options were examined in this work. Obviously, the results are case-dependent; they cannot necessarily be generalized for all SCOPF problems. However, for the specific test cases, our observations were consistent enough to indicate the more effective approaches. Detailed results are not presented for the sake of space.

3) Linearization: Proper power flow linearization constitutes a challenge on its own. As was observed in [21], which solved an OPF with all lines linearized, the problem is solved faster only for highly stressed systems; on average, the linearization slows down the solution process. Furthermore, convergence issues were observed in our simulations when linearizing too many lines, attributed to the voltage control constraints, (17)-(18), "clashing" with the large number of introduced voltage-angle constraints (see [21]). As such, only a percentage of power flows can be linearized, in order to avoid such cases. The linearization follows the same directive with the slack removal, i.e., it applies to lines that are thermally charged up to $80 \%$ of their upper limit (in the BC).

4) Network compression: The NC module comes with a major implementation issue: determining the proper number of equivalent buses for the inactive region. It is difficult to come up with an optimal bus number, as each contingency has different characteristics. A small number of equivalent buses (i.e., compressing loads/generators to a few load/generator centers) can be problematic, as the post-compression buses are associated with elements (loads, generators, impedances) of much larger values than normal buses. This creates scaling issues that significantly hinder convergence. Contrariwise, using too many equivalent buses results in negligible speed gains. Thus, finding a proper number of equivalent buses is not always easy. While not necessarily optimal, this work opts for an empirically sufficient compression, when the trade-off between 
TABLE IV: Solution algorithm results: 500-bus system (2 iterations required).

\begin{tabular}{|c|c|c|c|c|c|c|c|c|c|}
\hline & $\begin{array}{c}\text { Average CVE } \\
\text { time (s) }\end{array}$ & $\begin{array}{c}\text { Last SCOPF } \\
\text { time (s) }\end{array}$ & $\begin{array}{c}\text { Full solution } \\
\text { time (s) }\end{array}$ & $\begin{array}{c}\text { CCs added } \\
\text { (from 377) }\end{array}$ & $\begin{array}{c}\text { Gen. hitting } \\
\text { limits (from 62) }\end{array}$ & $\begin{array}{c}\text { Total } \\
\text { variables }\end{array}$ & $\begin{array}{c}\text { Total } \\
\text { constraints }\end{array}$ & Objective (\$) & Speed-up (\%) \\
\hline NLP & 10.13 & 205.13 & 350.64 & $8(2.12 \%)$ & $40(64.5 \%)$ & 10,116 & 20,792 & $49,274.8$ & - \\
NLP+LIN & 9.28 & 169.82 & 315.33 & $8(2.12 \%)$ & $40(64.5 \%)$ & 10,116 & 20,792 & $48,979.2$ & 10.09 \\
NLP+NC & 7.99 & 77.98 & 223.49 & $7(1.86 \%)$ & $36(58.1 \%)$ & 3,096 & 6,212 & $50,703.8$ & 36.25 \\
NLP+all & 7.13 & 60.55 & 170.06 & $7(1.86 \%)$ & $35(56.4 \%)$ & 3,096 & 6,212 & 50.700 .2 & 51.6 \\
\hline
\end{tabular}

TABLE V: Solution algorithm results: 2,000-bus system (3 iterations required).

\begin{tabular}{|c|c|c|c|c|c|c|c|c|c|}
\hline & $\begin{array}{c}\text { Average CVE } \\
\text { time (s) }\end{array}$ & $\begin{array}{c}\text { Last SCOPF } \\
\text { time (s) }\end{array}$ & $\begin{array}{c}\text { Full solution } \\
\text { time (s) }\end{array}$ & $\begin{array}{c}\text { CCs added } \\
\text { (from 3,200) }\end{array}$ & $\begin{array}{c}\text { Gen. hitting } \\
\text { limits (from 545) }\end{array}$ & $\begin{array}{c}\text { Total } \\
\text { variables }\end{array}$ & $\begin{array}{c}\text { Total } \\
\text { constraints }\end{array}$ & Objective (\$) & Speed-up (\%) \\
\hline NLP & 68.02 & 978.24 & $3,123.96$ & $14(0.44 \%)$ & $479(87.9 \%)$ & 35,630 & 79,340 & $908,293.6$ & - \\
NLP+LIN & 64.12 & 875.12 & $2,958.62$ & $14(0.44 \%)$ & $479(87.9 \%)$ & 35,630 & 79,340 & $901,935.5$ & 5.3 \\
NLP+NC & 52.10 & 462.67 & $2,300.04$ & $16(0.5 \%)$ & $441(80.9 \%)$ & 12,810 & 20,400 & $941,900.4$ & 26.38 \\
NLP+all & 44.13 & 414.52 & $1,969.55$ & $16(0.5 \%)$ & $439(80.5 \%)$ & 12,810 & 20,400 & $938,752.7$ & 39.64 \\
\hline
\end{tabular}

solution speed and objective value being deemed acceptable. Specifically, for the critical contingencies, the 500-bus system is compressed (on average) to 50 buses (90\% reduction) and the 2000-bus system is compressed (on average) to 170 buses (91.5\% reduction). While available, results for various other compression sizes are not presented for the sake of space.

\section{Results with different SCOPF versions}

The results of various versions of the SCOPF algorithm are presented in Tables IV, V. While for the 14-bus system we reach a solution in less than a second, the 500-bus system requires about 6 minutes to reach an acceptable solution and the 2000-bus system requires around 52 minutes. The SCOPF execution at the final iteration takes up the largest chunk of time (approximately 58.6\% and $31.3 \%$, respectively, for the two systems), while the CVE may also require some time to run for "problematic" contingencies (e.g., for the 2000-bus system, the average CVE takes over a minute to complete). Nonetheless, the ideal alternative, i.e., solving the full SCOPF problem, is not doable (computer memory exceeded) and impractical (there is minimal gain in considering every single $\mathrm{CC}$, as only a few of them are actually binding).

Initially (row 2), we linearize (for CCs) all lines whose $\mathrm{BC}$ power flow was less than $80 \%$ of their upper limit ( $\sim 70 \%$ of all lines). The linearization achieves a small speed improvement (on average, about $7.5 \%$ ) owing to the simplification of the CVE and SCOPF modules. However, the large number of introduced linear constraints (see [21]), coupled with the required voltage constraints, (17)-(18), does not allow for further improvement. The objective value is about $0.5 \%$ higher.

The effectiveness of NC is now separately tested (row 3). It achieves significant speed improvement (on average, about $31 \%$ ). For example, the speed improvement in the 2000bus system case is about a quarter of an hour (the time originally required by its largest SCOPF). This is due to the holistic simplification of the SCOPF module and the individual simplifications of the CVE modules, effectively eliminating more than $70 \%$ of all involved variables and constraints. The objective function value differs by approximately $2.9 \%$, mainly due to small line violations. In short, the NC module reduces the problem size significantly with small overal differences.
Lastly, NC is combined with linearization (linearization performed immediately after NC), as well as a favorable initialization (row 4). Compared to the original results, the speed improvement is significant. The time for the largest 500-bus SCOPF is reduced to about a minute (originally $\sim 3.5$ minutes), while the average time per CVE for the 2000-bus system is reduced by about $35 \%$. When one observes the total solution time, the effectiveness of the simplifications is more apparent, with the 2000-bus problem being solved in about 38 minutes (37\% speed improvement).

The NC module is certainly responsible for most of the speed improvement, reducing the problem size significantly. The linearization is also effective, further simplifying the problem structure of an already small network. While it is not easy to directly quantify the impact of each aspect on the solution speed-up, their synergy is undoubtedly successful. After all, the final speed-up is larger than the sum of speed-ups achieved by individual simplifications.

Each approximation plays its own part in solving the problem fast and efficiently. Their combination can achieve improved results, with each acting in a complementary manner to each other. This is an important observation, indicating the potential gains of treating all approximation in a collective manner instead of individually. Lastly, it is worth pointing out that the numbers obtained are actually not inapplicable; when the results (rows 1-4, Tables III, IV) are applied to the original formulation (as part of a validation PF), the obtained solution indicates that the problems are actually feasible.

A final comment must be made on interpreting the objective function value. For the 500-bus system, the slack penalties are nearly zero, while for the 2000-bus system only a few line slacks are (minimally) active, resulting in negligible penalties of less than $2 \%$ (as compared to the calculated objective value). This is because the penalty terms for constraints violations are set so high that, if slacks are activated, their value is kept very small. In other words, the majority of costs stems from the dispatch of generators, rather than from observed technical violations. Nonetheless, no generalization should be made at the moment, given the difficulty in ascertaining how case-specific the results are. The main takeaway is the 
employment of $\mathrm{NC}$ and linearization to render the problem manageable by solvers significantly speed up computations. Slight violations of some soft constraints might occur (as observed in similar studies), but only in post-contingency states. However, such solutions are usable for operators.

\section{CONClusions}

This paper proposed a combination of well-established and novel techniques for modelling and approximating securityconstrained optimal power flow (SCOPF) problems with practical industrial requirements, i.e., proper generator contingency response. These include an iterative NLP approximation of the original MINLP problem, the linearization of non-critical power flow constraints and the network compression of postcontingency states; the techniques can be used individually and in unison within an iterative algorithm of proven effectiveness to approximate SCOPF problems that are impossible to solve in reasonable times.

The proposed NLP approximation of generator responses works sufficiently well, with only minimal differences for a few generators and no voltage differences for PV buses. The complementary techniques, i.e., initialization, linearization and network compression (NC) all contributed to significant speed gains, with $\mathrm{NC}$ having the largest effect (about $60 \%$ of the speed improvement). The specific power flow linearization should be carefully employed, due to its potential of slowing down the solution or even leading to non-convergent cases. Proper initialization and heuristic slack removal can reduce the computational complexity and improving the solution speed.

\section{REFERENCES}

[1] B. Stott and O. Alsac. "Optimal power flow - basic requirements for real-life problems and their solutions" (White Paper). In SEPOPE XII Symposium, Brazil, May 2012.

[2] O. Alsac and B. Stott. "Optimal load flow with steady-state security". IEEE Trans. Power Apparatus, PAS-93(3):745-751, 1974.

[3] A.J. Monticelli, M.V.P. Pereira, and S. Granville. "Security-constrained optimal power flow with post-contingency corrective rescheduling". IEEE Trans. Power Syst., 2(1):175-182, 1987.

[4] F. Capitanescu, J. L. M. Ramos, P. Panciatici, D. Kirschen, A. M. Marcolini, L. Platbrood, and L. Wehenkel. "State-of-the-art, challenges, and future trends in security constrained optimal power flow". Electric Power Systems Research, 136(8):1731-1741, 2011.

[5] D. Phan and J. Kalagnanam. "Some Efficient Optimization Methods for Solving the Security-Constrained Optimal Power Flow Problem". IEEE Trans. Power Syst., 29(2):863-872, 2014.

[6] L. Platbrood, F. Capitanescu, C. Merckx, H. Crisciu, and L. Wehenkel. "A Generic Approach for Solving Nonlinear-Discrete SecurityConstrained Optimal Power Flow Problems in Large-Scale Systems". IEEE Trans. Power Systems, 29(3):1194-1203, 2013.

[7] J. Kardoš, D. Kourounis, and O. Schenk. "Two-Level Parallel Augmented Schur Complement Interior-Point Algorithms for the Solution of Security Constrained Optimal Power Flow Problems". IEEE Trans. Power Syst., 2019.

[8] O. Alsac, J. Bright, M. Prais, and B. Stott. "Further developments in LP-based optimal power flow". IEEE Trans. Power Syst., 2(5):697-711, 1990.

[9] Quanyuan Jiang, and Kai Xu. "A Novel Iterative Contingency Filtering Approach to Corrective Security-Constrained Optimal Power Flow". IEEE Trans. Power Syst., 29(3):1099-1109, 2014.

[10] E. Karangelos and L. Wehenkel. "An iterative AC-SCOPF approach managing the contingency and corrective control failure uncertainties with a probabilistic guarantee". IEEE Trans. Power Syst., 34(5):37803790, 2019.
[11] F. Capitanescu. "Critical review of recent advances and further developments needed in AC optimal power flow". Electric Power Systems Research, 136:57-68, 2016.

[12] A. D. Papalexopoulos, C. F. Imparato, and F. W. Wu. "Large-scale optimal power flow: effects of initialization, decoupling \& discretization". IEEE Trans. Power Syst., 4(2):748-759, 1989.

[13] W. Rosehart, C. Roman, and A. Schellenberg. "Optimal power flow with complementarity constraints". IEEE Trans. Power Syst., 20(2):813-822, 2005.

[14] K. Karoui, H. Crisciu, and L. Platbrood. "Modeling the primary reserve allocation in preventive and curative security constrained OPF". In IEEE PES T\&D, USA, 19-22 April 2010.

[15] Y. Dvorkin, P. Henneaux, D. Kirschen, and H. Pandžić. "Optimizing primary response in preventive security-constrained optimal power flow". IEEE Systems Journal, 12(1):414-423, 2016.

[16] F. Capitanescu. "Assessing reactive power reserves with respect to operating constraints and voltage stability". IEEE Trans. Power Syst., 26(4):2224-2234, 2011

[17] ARPA-E. SCOPF Problem Formulation: Challenge 1, Grid Optimization Competition, 2019. Available in http://www.gocompetition.energy.gov.

[18] B. Park, J. Holzer, and C.L. DeMarco. "A sparse tableau formulation for node-breaker representations in security-constrained optimal power flow". IEEE Trans. Power Syst., 34(1):637-647, 2018.

[19] L. de Magalhães Carvalho, A.M. Leite da Silva, and V. Miranda. "Security-Constrained Optimal Power Flow via Cross-Entropy Method". IEEE Trans. Power Syst., 33(6):6621-6629, 2018.

[20] S. M. Ashraf, B. Rathore, and S. Chakrabarti. "Performance analysis of static network reduction methods commonly used in power systems". In 18th National Power Systems Conference (NPSC), December 2014.

[21] D. Shchetinin, T. De Rubira, G. Hug. "On the Construction of Linear Approximations of Line Flow Constraints for AC Optimal Power Flow". IEEE Trans. Power Syst., 2(34):1182-1192, 2019.

[22] K. Karoui, H. Crisciu, A. Szekut, and M. Stubbe. "Large Scale Security Constrained Optimal Power Flow". In Proc. 16th Power Syst. Computation Conf., Glasgow, U.K., July 14-18, 2008.

[23] L. Platbrood, H. Crisciu, F. Capitanescu, and L. Wehenkel. "Solving very large scale SCOPF problems by combining iterative algorithms and network compression". In Proc. PSCC Conf., Sweden, August 2011.

[24] P. Dimo. Nodal analysis of power systems. Abacus Press, 1975.

[25] M. Gertz, J. Nocedal, and A. Sartenar. "A starting point strategy for nonlinear interior methods". Applied Mathematics Letter, 17(8):945952, 2004

[26] R. D. Zimmerman, C.E. Murillo-Sanchez, and R.J. Thomas. "MATPOWER: Steady-State Operations, Planning and Analysis Tools for Power Systems Research and Education". IEEE Trans. Power Syst., 26(1):12-19, 2011

[27] A. Wächter and L. T. Biegler. "On the Implementation of a Primal-Dual Interior Point Filter Line Search Algorithm for Large-Scale Nonlinear Programming". Mathematical Programming, 1(106):25-57, 2006.

[28] F. Capitanescu, M. Glavic, D. Ernst, and L. Wehenkel. "Contingency Filtering Techniques for Preventive Security-Constrained Optimal Power Flow". IEEE Trans. Power Syst., 22(4):1690-1697, 2007.

[29] P. Bonami, L.T. Biegler, A.R. Conn, et al. "An Algorithmic Framework for Convex Mixed Integer Nonlinear Programs". Discrete Optimization, 5(2):186-204, 2008.

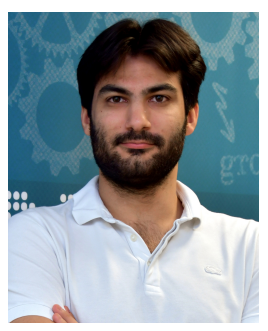

Iason-Iraklis Avramidis ( $\mathrm{S}$ ' 17) received the Dipl.Ing degree in Electrical and Computer Engineering from the National Technical University of Athens (NTUA), Greece, in 2017, and the M.Sc. degree in Energy Science and Technology from ETH Zurich, Switzerland, in 2019. He is currently with Environmental Research and Innovation (ERIN) at the Luxembourg Institute of Science and Technology (LIST), while pursuing the Ph.D. degree with the Electrical Energy \& Computer Architecture (ELECTA) Group, KU Leuven, Belgium. His research interests include the planning and operation of smart distribution grids, smart sustainable buildings and power markets. 
Florin Capitanescu received the Electrical Power Engineering degree from the University "Politehnica" of Bucharest, Romania, in 1997 and the Ph.D. degree from the University of Liège, Belgium, in 2003. Since 2015, he has been with ERIN at LIST as a researcher. His main research interests include the application of optimization methods to operation of transmission and active distribution systems, particularly optimal power flow approaches, voltage instability, and smart sustainable buildings.

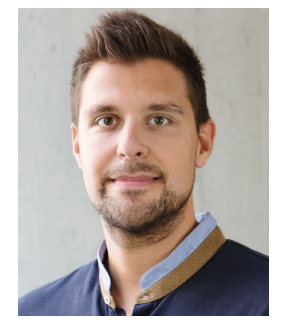

Stavros Karagiannopoulos (S'15-M'19) received a Diploma in Electrical and Computer Engineering from the Aristotle University of Thessaloniki, Greece, in 2010, and a M.Sc. degree in Energy Science and Technology from the Swiss Federal Institute of Technology (ETH), Zurich, Switzerland, in 2013. After his M.Sc., he worked at ABB Corporate Research Center in Switzerland until 2015. In 2019 he received the Ph.D. degree from the Swiss Federal Institute of Technology (ETH). Since August 2019, he has been working as a postdoctoral researcher with Power Systems Laboratory at ETH Zurich, and since 2020 also with the Laboratory for Information and Decision Systems at Massachusetts Institute of Technology (MIT). His main research focuses on data-driven and optimization-based methods for active distribution grids.

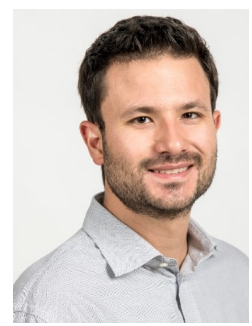

Evangelos Vrettos (S'09-M'16) is a Research and Digitalization Manager at Swissgrid AG. Prior to that, he was a Research Scientist at Lawrence Berkeley National Laboratory (Grid Integration Group) and a Home Energy Systems Engineer at the Smart Grid Laboratory of the Swiss electric utility EKZ (Elektrizitätswerke des Kantons Zürich). He received the Dipl.-Ing degree in Electrical and Computer Engineering from the National Technical University of Athens (NTUA), Greece, in 2010. After his graduation, he worked as a research assistant at the Electric Machines and Power Electronics Laboratory of NTUA. In 2011, he joined the Power Systems Laboratory of ETH Zurich, where he obtained the Ph.D. degree in Electrical Engineering and Information Technology in 2016 and continued as a postdoctoral researcher before joining EKZ. His current research interests include demand-side management for renewable energy integration, optimal power flow applications for congestion management, and control of power grids with low rotational inertia. 